\title{
Use of silicone stents for the management of post-tuberculosis tracheobronchial
}

\section{stenosis}

\author{
Y.J. Ryu*, H. Kim", C.M. Yü, J.C. Choi ${ }^{\#}$, Y.S. Kwon" and O.J. Kwon ${ }^{\#}$
}

ABSTRACT: The role of bronchoscopic management in post-tuberculosis tracheobronchial stenosis is not well defined. To investigate the role of bronchoscopic intervention, including silicone stenting, in the management of post-tuberculosis tracheobronchial stenosis, the current retrospective study was conducted at a tertiary referral hospital.

Under rigid bronchoscopy, 80 patients underwent ballooning, neodymium-yttrium aluminium garnet laser resection and/or bougienation as first-line methods of airway dilatation between January 2000 and December 2003 inclusive, and were followed for a median of 41 months.

Silicone stents were required in 75 out of 80 (94\%) patients to maintain airway patency. Bronchoscopic intervention provided immediate symptomatic relief and improved lung function in $\mathbf{8 8} \%$ of the patients. After airway stabilisation, stents were removed successfully in 49 out of 75 (65\%) patients at a median of 14 months post-insertion. Three patients out of 75 (4\%) eventually underwent surgical management. Acute complications included: excessive bleeding $(n=1)$; pneumothorax $(n=5)$; and pneumomediastinum without mortality $(n=2)$. Stent-related late complications, such as migration (51\%), granuloma formation (49\%), mucostasis (19\%) and restenosis $(40 \%)$, were controllable during a median follow-up of 41 months.

In conclusion, bronchoscopic intervention, including silicone stenting, could be a useful and safe method for treating post-tuberculosis tracheobronchial stenosis.

\section{KEYWORDS: Airway stenoses, bronchoscopy, intervention, tuberculosis}

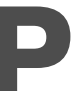
ost-tuberculosis tracheobronchial stenosis (PTTS) is the most common cause of benign tracheobronchial stenosis in tuberculosis (TB) endemic areas, such as South Korea, where the prevalence of pulmonary TB is $1.0 \%$ [1, 2]. Even when taking effective anti-TB medication, some cases of PTTS may progress to obstruction of major airways [1, 2]. Surgical resection and reconstruction after the eradication of Mycobacterium tuberculosis has been the preferred treatment for most patients with PTTS [1, 2].

Stenting has been developed to deal with airway stenosis and avoid the potential morbidities of open surgery. However, the use of metallic stents has resulted in some irreversible complications, such as stent fracture and impaction into the mediastinum [3, 4]. Interventional bronchology and silicone airway stenting has opened up a new way to treat patients with benign airway stenosis $[3,4]$. Until recently, only a few studies have reported the result of bronchoscopic intervention in patients with PTTS [5-8]. However, the number of patients was $<40$ in some of these.
The current authors have experienced 80 cases of PTTS requiring bronchoscopic intervention, including ballooning, laser resection and/or silicone stenting. The purpose of the present study was to investigate the clinical efficacy of bronchoscopic intervention with the role of silicone stenting in the management of PTTS patients.

\section{PATIENTS AND METHODS}

\section{Inclusion criteria}

In total, 80 patients who underwent bronchoscopic intervention for the treatment of PTTS at the Samsung Medical Center (Seoul, South Korea) were included between January 2000 and December 2003. To describe clinical characteristics, the methods of management used and the clinical outcomes achieved, medical records were retrospectively reviewed.

Bronchoscopic intervention in patients with PTTS diagnosed by three-dimensional computed tomography scans and/or bronchoscopy were indicated by the following conditions: 1) a

\section{AFFILIATIONS}

*Division of Pulmonary and Critical Care Medicine, Dept of Internal Medicine, Dongdaemun Hospital, Ewha Womans University, College of Medicine, and

\#Division of Pulmonary and Critical Care Medicine, Dept of Medicine, Samsung Medical Center,

Sungkyunkwan University School of Medicine, Seoul, Republic of Korea.

\section{CORRESPONDENCE}

H. Kim

Division of Pulmonary and Critical

Care Medicine

Dept of Medicine

Samsung Medical Center

Sungkyunkwan University School of Medicine

50 Irwon-dong

Gangnam-gu

Seoul

$135-710$

Republic of Korea

Fax: 82234103849

E-mail: hjkim@smc.samsung.co.kr

Received:

February 102006

Accepted after revision:

August 162006

\section{SUPPORT STATEMENT}

This work was supported by a Korean Health \& Welfare grant, Republic of Korea (00-PJI-PG1-CH12-0006). 
progressive deterioration of respiratory symptoms, such as progressive dyspnoea; or 2 ) recurrent ( $>2$ yrs) post-obstructive pneumonia; or 3) an abrupt complete collapse of one lung with reduced lung function.

For patients with active $\mathrm{TB}$, adequate medications were administered according to American Thoracic Society guidelines $[9,10]$. In most patients with active TB, bronchoscopic intervention was postponed until negative conversion. However, when complete lung collapse occurred or lifethreatening dyspnoea developed, intervention was performed regardless of activity.

\section{Definitions and parameters}

Active TB was defined as the documentation of acid-fast bacilli by staining or culture for $M$. tuberculosis from sputum or bronchial aspirate and/or bronchial biopsy. Previous TB was defined as a definite history of $M$. tuberculosis infection proven by microbiology or pathology.

Forced expiratory volume in one second (FEV1) was used as an objective parameter of ventilation. FEV1 could be obtained in 76 patients before the intervention and in 73 patients just after intervention.

\section{Stents}

In 2002, a newly designed silicone stent known as the Natural stent was developed at the Samsung Medical Center (fig. 1) [11]. This stent is composed of moulded silicone and is straight in shape. It features regularly placed " $\mathrm{C}$ " circular studs on its outer surface by interposing a flexible posterior wall, which mimics the posterior membrane of the trachea for the tracheal stenosis, or external round studs without an interposing smooth wall for bronchial stenosis. These stent designs increase stent-to-wall contact and maintain physiological airway constriction and dilation. Previous studies in a canine model of tracheal stenosis and clinical experience in patients with benign tracheobronchial stenosis have shown that the Natural stent is as effective and safe as the Dumon stent $[11,12]$.

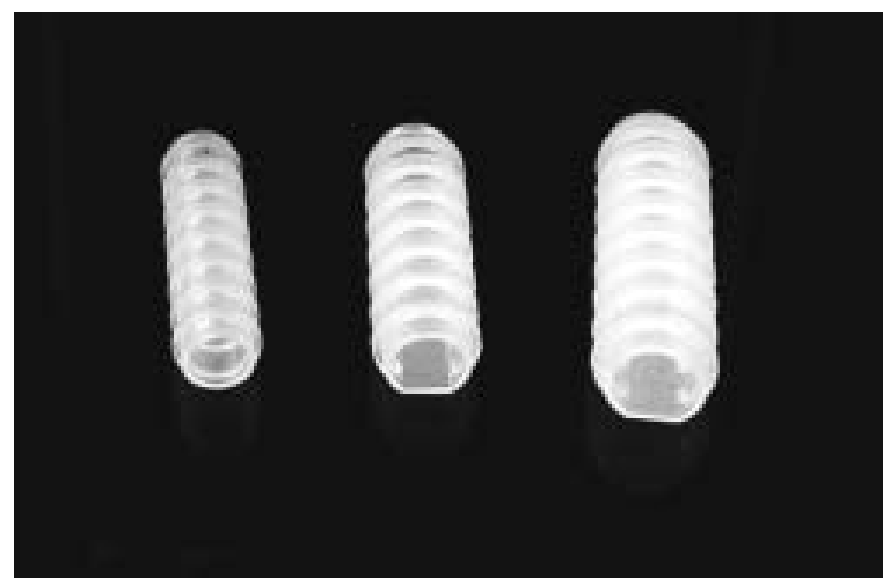

FIGURE 1. Three Natural silicone stents. On the left is a 10-mm stent for the bronchus. In the middle is a 12-mm stent for a female trachea, and on the right a 14$\mathrm{mm}$ stent for a male trachea.
A Dumon stent (BryanCorp, Woburn, MA, USA) or a Natural stent (TNO Co., Seoul, South Korea) of 12-14-mm outer diameter was used for tracheal stenosis, and a $10-\mathrm{mm}$ stent for bronchial stenosis. Adequate size and type of stent was selected and used according to the interventionist's decision. The use of the Natural silicone stents in the current study was approved by the Institutional Review Board at the Samsung Medical Center and written informed consent was obtained from all participants.

\section{Airway intervention techniques}

Airway intervention was performed following the standard techniques of COLT and DUMON [3] and KiM [4]. After the induction of general anaesthesia, patients were intubated with a rigid bronchoscope tube (Hopkins, Karl-Storz, Tuttlingen, Germany). A flexible bronchoscope (EVIS BF 1T240; Olympus, Tokyo, Japan) was then introduced through the rigid bronchoscope, and airway narrowing was evaluated. For ballooning, a $10 \mathrm{~mm}$-sized controlled radial-expansion balloon (Boston Scientific, Boston, MA, USA) was inflated two or three times to $303.9 \mathrm{kPa}$ for $20 \mathrm{~s}$. When localised dense fibrosis was observed, a neodymium-yttrium aluminium garnet laser (LaserSonics, Milpitas, CA, USA) was used to cut fibrotic bands, using a G56D noncontact fibre (LaserSonics). Fibrotic stenoses were also gently dilated mechanically using a rigid bronchoscope. Mechanical dilatation was immediately followed by stent insertion. A stent of an appropriate size was folded longitudinally, introduced into a stent pusher (BryanCorp), and properly re-positioned using alligator forceps. Airway stents were implanted using the standard technique described by DUMON [13]. Silicone stenting was indicated when: 1) malacia of $>180^{\circ}$ of the angle of the dilated lumen was observed; 2 ) the longitudinal stenotic segment was longer than $2 \mathrm{~cm}$; or 3) a third recurrence of airway narrowing after the intervention occurred.

After airway intervention, patients were usually discharged from hospital $24 \mathrm{~h}$ after the procedure and followed up with chest radiography and spirometry 1, 3, 6, 9 and 12 months after intervention, and every 6 months thereafter. All patients were followed for at least 24 months.

\section{Statistical analysis}

A p-value $<0.05$ was considered significant. Group comparisons of categorical variables were made using the Pearson Chi-squared test or Fisher's exact test. To assess relationships between the groups' continuous variables, the Mann-Whitney U-test was used for nonnormally distributed data or the Wilcoxon signed-ranks test for paired FEV1 data. Values are expressed as median (range) values for continuous variables, or as numbers (percentages).

\section{RESULTS}

The characteristics of study participants are summarised in table 1 . There was a predominance of females, with a median (range) age of 33 (14-73) yrs. The most common location of stenosis was the left main bronchus (65\%).

\section{Bronchoscopic findings and interventions}

The bronchoscopic findings and interventions are summarised in table 2. A representative case is presented in figure 2. 
TABLE 1 Baseline characteristics of the study population

\section{Characteristics}

\begin{tabular}{lc} 
Subjects & 80 \\
Sex male/female & $9 / 71$ \\
Age yrs & $33(14-73)$ \\
Disease activity on first intervention ${ }^{\#}$ & \\
Active tuberculosis & $33(41)$ \\
Past tuberculosis & $47(59)$ \\
Chest radiography & \\
No abnormal parenchymal lesion & $9(11)$ \\
Atelectasis or collapse & $40(50)$ \\
Consolidation with volume loss & $14(18)$ \\
Fibrous lesion \pm calcified granuloma & $17(21)$ \\
Baseline pulmonary function test & \\
FEV1 \% pred & $63(15-100)$ \\
FVC \% pred & $73(40-102)$ \\
FEV1/FVC ratio & $69(17-97)$ \\
Sites of stenosis & 86 \\
Trachea & $12(14)$ \\
Left main bronchus & $56(65)$ \\
Right main bronchus & $11(13)$ \\
Bronchus intermedius & $6(7)$ \\
Left lower lobar bronchus & $1(1)$ \\
\hline
\end{tabular}

Data are presented as $\mathrm{n}$, median (range) or $\mathrm{n}(\%)$. FEV 1 : forced expiratory volume in one second; \% pred: percentage predicted; FVC: forced vital capacity. ${ }^{\#}$ : either bacteriological or pathological; " : overlapped.

The median (range) luminal diameter was $9.5(8-11) \mathrm{mm}$ in the trachea and 5 (4-6) $\mathrm{mm}$ in the bronchus when the lumen was measured by bronchoscopy.

The most common method of airway dilation was ballooning in 77 out of 109 patients. However, airway dilatation was usually performed in combined modalities. After airway dilation, stenting was needed in 75 out of 80 (93.7\%) patients.

\section{Outcomes and complications}

Overall clinical outcomes are summarised in table 3 and figure 3. Immediate subjective relief of dyspnoea was achieved in 70 out of $80(88 \%)$ patients. After the intervention, the median luminal diameter was increased from 9.5 to $10 \mathrm{~mm}$ in the trachea and from 5 to $7 \mathrm{~mm}$ in the bronchus.

Spirometry was repeated 1 month after the intervention. The FEV1 \% predicted significantly improved by a median (range) of 21.8 (-9-258) \% in each patient. After the stent was removed, improved FEV1 \% pred was maintained (fig. 4).

The median (range) duration of overall stent placement in all 75 patients was $24(1-50)$ months. Stents could be removed in 54 out of $75(72 \%)$ patients after a median of 14 months. In 54 patients in whom a stent was removed, 49 patients had a successful stable clinical course during a follow-up period of 36 months. However, five patients showed recurrence and needed re-stenting. Finally, 23 (18 plus five recurred) out of 75 patients $(31 \%)$ experienced prolonged stent placement of a median (range) 32 (1-55) months as shown in figure 3 . Three patients showed no response after intervention and underwent

\begin{tabular}{|c|c|c|}
\hline TABLE 2 & \multicolumn{2}{|c|}{$\begin{array}{l}\text { Bronchoscopic findings and parameters of } \\
\text { intervention }\end{array}$} \\
\hline \multicolumn{3}{|c|}{ Stenosis type ${ }^{\#}$} \\
\hline Fibrous st & ture & $55(69)$ \\
\hline Malacia & & $8(10)$ \\
\hline Fibrous st & ture and malacia & $17(21)$ \\
\hline \multicolumn{3}{|c|}{ Luminal diameter } \\
\hline Trachea $\mathrm{m}$ & & $9.5(8-11)$ \\
\hline Main bron & us $\mathrm{mm}^{+}$ & $5(4-6)$ \\
\hline \multicolumn{3}{|c|}{ Time of intervention } \\
\hline Active TB & er starting anti-TB therapy months ${ }^{\S}$ & $6(1-11)$ \\
\hline Past TB a & r starting anti-TB therapy yrs ${ }^{f}$ & $5(1-40)$ \\
\hline \multicolumn{3}{|c|}{ Method of airway dilatation overlapped $\# \#$} \\
\hline Ballooning & & 77 \\
\hline Nd-YAG Ia & & 13 \\
\hline Bougienat & & 19 \\
\hline Number of & sed stent per patient & $2(1-12)$ \\
\hline Duration of & verall stent placement months" & $24(3-50)$ \\
\hline Duration of & llow-up months ${ }^{++}$ & $41(24-53)$ \\
\hline
\end{tabular}

surgical resection involving end-to-end anastomosis. During a median (range) follow-up period of 41 (24-53) months, clinical outcome was unchanged in all patients.

The majority of patients experienced minor complications, which included cough, mucus plugging and blood-tinged sputum; however, stents were tolerable. Eight out of $80(10 \%)$ patients experienced major complications. Massive bleeding developed in one patient, and the patient was treated by mechanical ventilation and blood transfusions without mortality. Pneumothorax occurred in five patients and pneumomediastinum in two patients. These air leakage complications were treated by oxygen supplement and/or chest tube insertion in three patients.

\begin{tabular}{|c|c|c|}
\hline TABLE 3 & \multicolumn{2}{|c|}{$\begin{array}{l}\text { Outcome of bronchoscopic intervention in } \\
\text { patients with post-tuberculosis tracheobronchial } \\
\text { stenosis }\end{array}$} \\
\hline \multicolumn{3}{|c|}{ Immediate symptomatic relief ${ }^{\#}$} \\
\hline Improved & & $70(88)$ \\
\hline Unchanged & & $10(12)$ \\
\hline \multicolumn{2}{|c|}{ Rate of successful stent removal } & $65(49 / 75)$ \\
\hline \multicolumn{2}{|c|}{ Duration of stent placement months } & $14(1-47)$ \\
\hline \multicolumn{2}{|c|}{ Follow-up after removal months } & $36(3-50)$ \\
\hline \multicolumn{2}{|c|}{ Rate of persistent stent placement } & $31(23 / 75)$ \\
\hline \multicolumn{2}{|c|}{ Duration of stent placement months } & $32(1-55)$ \\
\hline \multicolumn{2}{|c|}{ Rate of surgical management } & $4(3 / 75)$ \\
\hline \multicolumn{2}{|c|}{ Follow-up after surgery months } & $30(21-38)$ \\
\hline
\end{tabular}

Data are presented as $n(\%), \%(n / N)$ or median (range). ${ }^{*}: n=80 ; "$ : these patients underwent surgical resection with end-to-end anastomosis. 

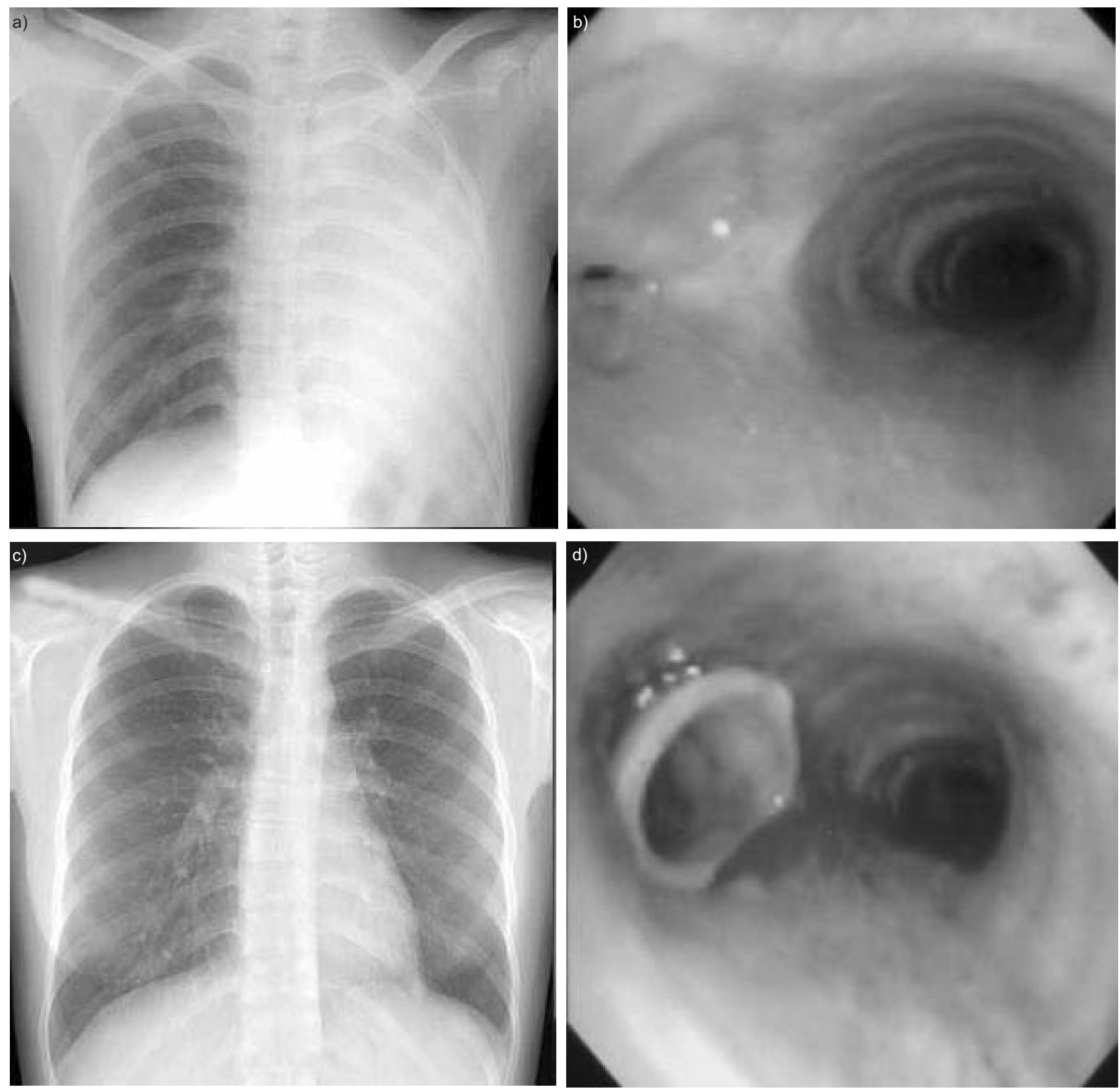

FIGURE 2. A representative case of bronchial intervention. Marked narrowing of the left main bronchus was noted in a 32-yr-old female patient (a, b). After the 10-mm silicone stent was inserted, the left main bronchus was widened to normal calibre (c, d).

Stent-related late complications were common. Stent migration developed in 38 out of $75(51 \%)$ patients, in growth of granulation tissue at the end of the stent or through stent interstices occurred in 37 of the $75(49 \%)$ patients, and restenosis in $30(40 \%)$ patients. Repeated bronchoscopic interventions were needed to treat complications. The median (range) number of bronchoscopic interventions was 4 (1-16) and the median (range) number of stents used was 2 (1-12) per patient during a median follow-up of 41 months.

\section{Subgroup analysis}

Patients were subgrouped into those who had the stent successfully removed (stent removed, table 4) and those who could not have it removed (stent remaining, table 4). Patients in whom the stent could not be removed or re-inserted (stent remaining) were found to have a lower baseline FEV1 and needed bougienation more frequently for pre-stenting dilatation. Restenosis and granulation tissue formation were also more frequently observed in patients in whom the stent was remaining. 


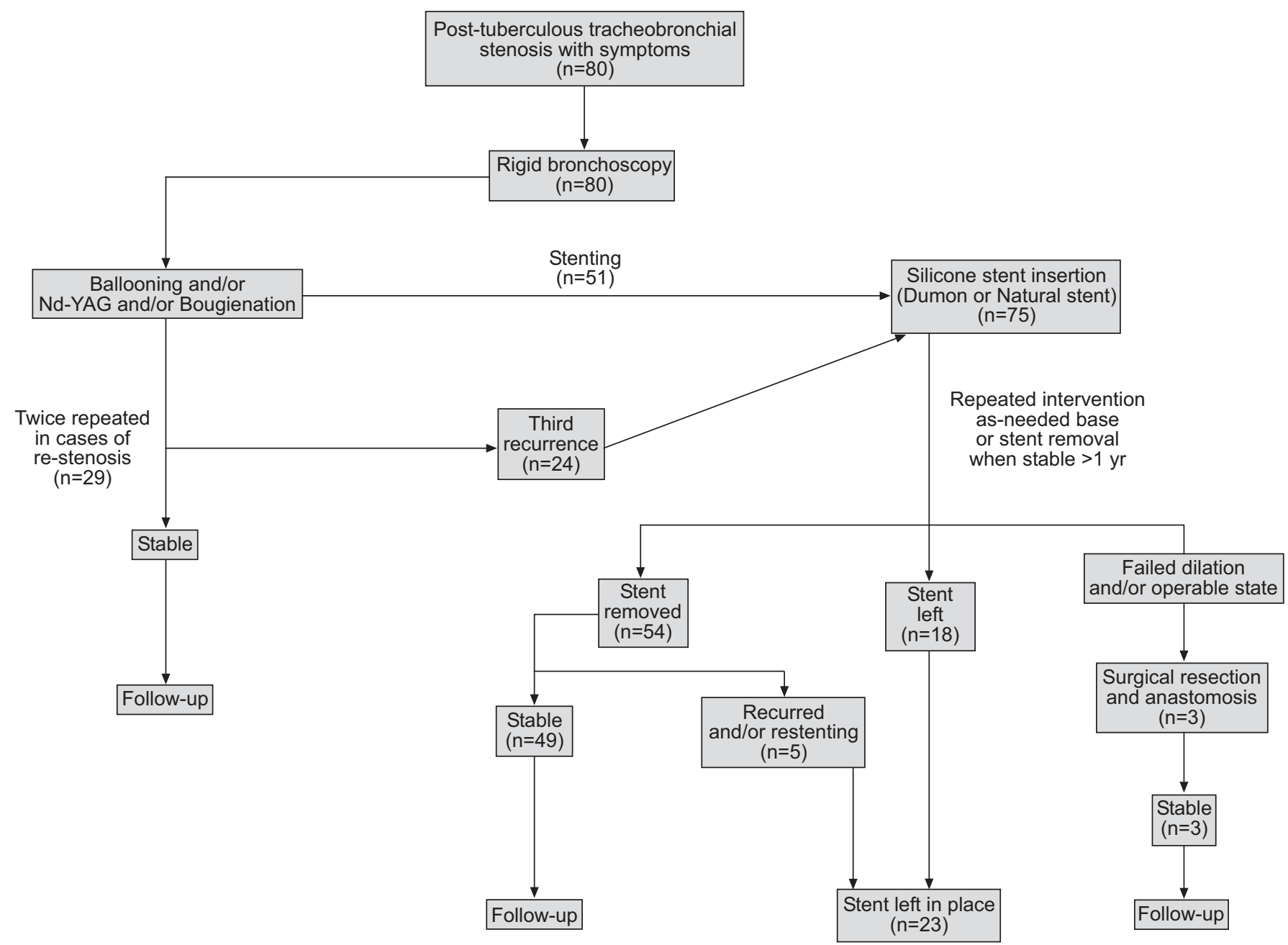

FIGURE 3. Therapeutic diagram of bronchoscopic interventions in 80 post-tuberculosis tracheobronchial stenosis patients. Nd-YAG: neodymium-yttrium aluminium garnet.

Subgroup analysis was also performed according to the type of stent. No significant difference was found with respect to sex ratio, median age, TB activity, or sites of tracheobronchial stenosis between those that received a Dumon or a Natural stent (data not shown). Moreover, no significant difference was found in clinical outcomes and complications between the types of stent.

When clinical outcomes were compared for patients with active and past TB, re-stenosis was more frequently developed in patients with active TB (63 versus $37 \%, p=0.005)$, but no significant difference was found for the other clinical parameters.

\section{DISCUSSION}

The present study demonstrates that bronchoscopic intervention, including silicone stenting, could be a useful method for treating patients with PTTS. To the current authors' knowledge, the present study is the largest series of PTTS managed by bronchoscopic intervention with silicone stenting.

Airway involvement by respiratory tract $\mathrm{TB}$ was reported to occur in $11-15 \%$ of routine bronchoscopies in a TB sanatorium [14]. After effective anti-TB chemotherapy, endotracheal or endobronchial TB usually responds well and, in particular, generally little subclinical airway narrowing remains. However, in patients with symptomatic airway stenosis, bronchoscopic intervention is essential for improving lung function and controlling TB.

Studies have been conducted on bronchoscopic intervention using ballooning and stenting [5-8, 12, 15]. However, recurrence is common in patients who have undergone balloon dilatation. In the present study, ballooning was successful in only five out of $80(6.3 \%)$ PTTS patients, and airway stenting was required in the majority of patients (75 out of $80,93.7 \%$ ). As granulomatous inflammation and fibrosis are natural consequences of the TB healing process, mechanical dilation alone might be ineffective to maintain airway patency.

Metallic stents have been used in PTTS patients, but critical complications have been reported [5-8, 12, 15]. Therefore, removable silicone stents, such as Dumon stents, are preferred for benign airway stenoses, especially in PTTS patients [5-8, 12, 15]. Silicone stents have the advantage that they can be repositioned and removed as many times as needed. In PTTS patients, airway remodelling usually follows bronchoscopic intervention. Therefore, stent re-position, and even stent 


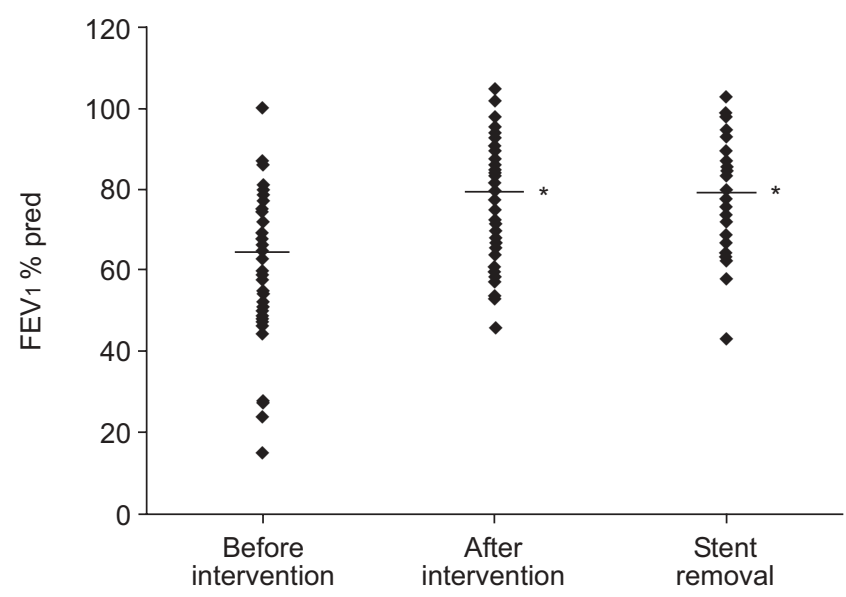

FIGURE 4. Forced expiratory volume in one second (FEV 1$)$ before $(n=76)$ and after intervention $(n=73)$, and after stent removal $(n=34)$. \% pred: per cent predicted. Horizontal bars represent the median value. *: $p<0.05$ compared with FEV1 before intervention.

changes, are necessary to manage frequent stent migration and airway re-stenosis. In the present study, stent migration was observed in 38 out of 75 (51\%) patients and re-stenosis in 30 $(40 \%)$ patients. These complication rates are higher than previous studies, in which most of the included patients were non-PTTS [16-20].

Although no definite guidelines have been issued concerning the timing of stent removal, previous studies have recommended that in cases of benign airway stenosis stents should be placed for $6-18$ months $[13,16]$. In the present study, stents could be successfully removed a median of 14 months after insertion in 49 out of the 75 (65\%) patients. These patients then showed a stable clinical course for a median of 36 months. Considering the natural fibrotic process that occurs after endobronchial TB by $M$. tuberculosis infection [2, 21], stents should be replaced at least once a year.

In 23 out of the 75 (31\%) patients, stents could not be removed due to recurrent airway stenosis or malacia. When the group of successful stent removal was compared with those of stent remaining, there was more frequent granulation tissue formation or re-stenosis by fibrotic stricture. Extensive fibrosis could provide an explanation for these patients because they showed reduced baseline pulmonary function and needed bougienation more frequently due to tight stricture.

Fibrotic stricture of the airway in PTTS is usually eccentric. As the fibrotic lesion is composed of dense fibrosis tissue, it would be more resistant to the stretching tension. When balloon or bougie is blindly used, the fibrotic stricture would not be widened and only the cartilage of normal airway would be injured resulting in the aggravation of airway malacia. Therefore, the use of a laser or mechanical dilatation on the direct vision of the stricture should be encouraged rather than blind ballooning in patients with PTTS.

Re-stenosis was more frequent in patients with active TB than in those with past TB. Granulation tissue formation and fibrosis are natural host defence mechanisms against TB by anatomical isolation of infection. As these defence mechanisms

\begin{tabular}{|c|c|c|c|}
\hline $\begin{array}{l}\text { Subgroup a } \\
\text { stent was re }\end{array}$ & $\begin{array}{l}\text { s between } \\
\text { d or remal }\end{array}$ & $\begin{array}{l}\text { atients in } \\
\text { ing }\end{array}$ & hom \\
\hline Variables & $\begin{array}{c}\text { Stent } \\
\text { removed }\end{array}$ & $\begin{array}{c}\text { Stent } \\
\text { remaining }\end{array}$ & p-value \\
\hline Subjects & 49 & 23 & \\
\hline Sex male/female & $8 / 41$ & $1 / 22$ & 0.255 \\
\hline Age yrs & $34(17-73)$ & $29(14-50)$ & 0.060 \\
\hline Active/past TB & $18 / 31$ & $13 / 10$ & 0.114 \\
\hline \multicolumn{4}{|l|}{ FEV1 \% pred } \\
\hline Before stenting & $65(24-100)$ & $55(15-78)$ & 0.013 \\
\hline After stenting & $79(46-117)$ & $73(34-105)$ & 0.184 \\
\hline Change after stenting $\#$ \% & $19(-9-258)$ & $28(-1-159)$ & 0.159 \\
\hline Tracheal involvement & $5(10)$ & $5(22)$ & 0.273 \\
\hline Stenosis type & & & 0.555 \\
\hline Fibrous stricture & $32(65)$ & $17(74)$ & \\
\hline Malacia & $6(12)$ & $1(4)$ & \\
\hline Fibrous stricture and malacia & $11(22)$ & $5(22)$ & \\
\hline \multicolumn{4}{|l|}{ Method of airway dilatation } \\
\hline Ballooning & $47(96)$ & $22(96)$ & 1.000 \\
\hline Nd-YAG laser & $7(14)$ & $4(17)$ & 0.736 \\
\hline Bougienation & $8(16)$ & $9(39)$ & 0.034 \\
\hline Type of stent used & & & 0.258 \\
\hline Dumon & $24(49)$ & $8(35)$ & \\
\hline Natural & $25(51)$ & $15(65)$ & \\
\hline \multicolumn{4}{|l|}{ Late complications } \\
\hline Stent migration & $23(47)$ & $13(5 \%)$ & 0.251 \\
\hline Granulation tissue formation & $20(41)$ & $14(61)$ & 0.047 \\
\hline Re-stenosis & $13(27)$ & $15(65)$ & $<0.0001$ \\
\hline
\end{tabular}

Data are presented as $n$, median (range) or $n$ (\%). TB: tuberculosis; FEV1: forced expiratory volume in one second; \% pred: percentage predicted; $\mathrm{Nd}$ YAG: neodymium-yttrium aluminium garnet. * : (FEV1 \% before stenting-after stenting)/FEV $1 \%$ before stenting $\times 100 \%$.

are ongoing, re-stenosis would be more frequent in patients with active TB. In addition, a paradoxical aggravation of airway TB may occur, especially in patients with endotracheal TB. Although adequate anti-TB medications have been given, the current authors experienced that active endotracheal TB spread longitudinally over the stented trachea. These patients required a longer stent or another stenting. However, almost all of these patients could be treated by prolonged anti-TB medications and repeated intervention.

No difference was found according to the type of stent, i.e. Dumon or Natural stents. The present study suggests that Natural stents could be an alternative to Dumon stents in patients with PTTS, but, to confirm this, a prospective randomised study is required.

In conclusion, it was found that bronchoscopic intervention with silicone stenting could provide an effective means of managing post-tuberculosis tracheobronchial stenosis patients.

\section{REFERENCES}

1 Chung HS, Lee JH. Bronchoscopic assessment of the evolution of endobronchial tuberculosis. Chest 2000; 117: 385-392. 
2 Kim YH, Kim HT, Lee KS, Uh ST, Cung YT, Park CS. Serial fiberoptic bronchoscopic observations of endobronchial tuberculosis before and early after antituberculosis chemotherapy. Chest 1993; 103: 673-677.

3 Colt HG, Dumon JF. Airway stents. Present and future. Clin Chest Med 1995; 16: 465-478.

$4 \mathrm{Kim} \mathrm{H}$. Stenting therapy for stenosing airway disease. Respirology 1998; 3: 221-228.

5 Watanabe Y, Murakami S, Oda M, et al. Treatment of bronchial stricture due to endobronchial tuberculosis. World J Surg 1997; 21: 480-487.

6 Nomori H, Horio H, Suemasu K. Granulation stenosis caused by a Dumon stent placed for endobronchial tuberculous stenosis. Surg Laparosc Endosc Percutan Tech 2000; 10: 41-43.

7 Iwamoto Y, Miyazawa T, Kurimoto N, et al. Interventional bronchoscopy in the management of airway stenosis due to tracheobronchial tuberculosis. Chest 2004; 126: 1344-1352.

8 Low SY, Hsu A, Eng P. Interventional bronchoscopy for tuberculous tracheobronchial stenosis. Eur Respir J 2004; 24: 345-347.

9 Bass JB Jr, Farer LS, Hopewell PC, et al. Treatment of tuberculosis and tuberculosis infection in adults and children. American Thoracic Society and The Centers for Disease Control and Prevention. Am J Respir Crit Care Med 1994; 149: 1359-1374.

10 Blumberg HM, Burman WJ, Chaisson RE, et al. American Thoracic Society/Centers for Disease Control and Prevention/Infectious Diseases Society of America: treatment of tuberculosis. Am J Respir Crit Care Med 2003; 167: 603-662.

$11 \mathrm{Kim} \mathrm{H}, \mathrm{Koh} W$, Suh G, et al. The useful and safety of Natural stent in a canine model of tracheal stenosis. Tuberculosis Respir Dis 2002; 53: 431-438.
12 Ryu YJ, Yu CM, Choi JC, et al. Clinical experience of silicone airway stent in the management of benign tracheobronchial stenosis. Tuberculosis Respir Dis 2005; 59: 62-68.

13 Dumon JF. A dedicated tracheobronchial stent. Chest 1990; 97: 328-332.

14 Mac RD, Hiltz JE, Quinlan JJ. Bronchoscopy in a sanatorium; a review of 522 consecutive bronchoscopies. Am Rev Tuberc 1950; 61: 355-368.

15 Lee SS, Kim DH, Baik HC, Lee DY. Clinical analysis of expandable metallic stent in benign tracheal and bronchial disease. Korean J Bronchoesophagol 2004; 10: 17-21.

16 Martinez-Ballarin JI, Diaz-Jimenez JP, Castro MJ, Moya JA. Silicone stents in the management of benign tracheobronchial stenoses. Tolerance and early results in 63 patients. Chest 1996; 109: 626-629.

17 Puma F, Ragusa M, Avenia N, et al. The role of silicone stents in the treatment of cicatricial tracheal stenoses. $J$ Thorac Cardiovasc Surg 2000; 120: 1064-1069.

18 Bolliger CT, Probst R, Tschopp K, Soler M, Perruchoud AP. Silicone stents in the management of inoperable tracheobronchial stenoses. Indications and limitations. Chest 1993; 104: 1653-1659.

19 Abdullah V, Yim AP, Wormald PJ, van Hasselt CA. Dumon silicone stents in obstructive tracheobronchial lesions: the Hong Kong experience. Otolaryngol Head Neck Surg 1998; 118: 256-260.

20 Noppen M, Meysman M, Claes I, D'Haese J, Vincken W. Screw-thread versus Dumon endoprosthesis in the management of tracheal stenosis. Chest 1999; 115: 532-535.

21 Lee JH, Chung HS. Bronchoscopic, radiologic and pulmonary function evaluation of endobronchial tuberculosis. Respirology 2000; 5: 411-417. 